\title{
Mapping the Field Distribution of a Photonic Crystal Resonator, using Transmission SNOM
}

\author{
Wico C.L. Hopman, Kees O. van der Werf, René M. de Ridder \\ University of Twente, MESA + Institute for Nanotechnology, PO Box 217, 7500 AE Enschede, The Netherlands, \\ Phone: +31 (0)53489 4440, Fax:+31 (0)534893343. \\ Email address: W.C.L.Hopman@utwente.nl
}

\begin{abstract}
We show that transmission SNOM (T-SNOM) can be used for imaging optical intensity distributions in a photonic crystal resonator with a resolution better than $100 \mathrm{~nm}$. Two alternatives are explored: contact and tapping mode operation. (C)2007 Optical Society of America OCIS codes: (180.5810) Scanning microscopy; (170.4520) Optical confinement and manipulation; (130.3120) Integrated optics devices; (120.7000) Transmission; (140.4780) Optical resonators.
\end{abstract}

\section{Introduction}

Existing scanning near-field optical microscopy (SNOM) can be used to map the resonance wave patterns in highindex microcavities with resolution below the diffraction limit. However, the interpretation of these results is involved, because the measurements interfere with the device operation. In, for example, photonic scanning tunneling microscopy (PSTM), the roughly $100 \mathrm{~nm}$ radius metal coated fiber tip interferes with the field inside the resonator, altering the original properties like the quality factor $Q$ and resonance wavelength. A similar argument can be found for scattering based SNOM. In particular, this can be a problem with high- $Q$ resonators which can be considerably detuned by the presence of a nano-sized probe [1,2].

Recently a novel SNOM method has been proposed: transmission SNOM or T-SNOM [2,3]. The transmitted (or reflected) light is monitored, while the evanescent field is perturbed by scanning a nano-sized probe over the resonator. The advantage compared to the other SNOM methods are that the scanning tip can be significantly smaller (potentially increasing the resolution) without necessarily decreasing the sensitivity. Furthermore, there is a large freedom in choosing the tip material, allowing selecting the strength of the mechano-optical interaction. In [4], we showed that the presence of a dielectric probe can give rise to a change in transmission if the cavity is tuned near the resonance wavelength. If the spectral shape of the resonance is preserved, i.e. the amount of induced out of plane scattering is low, the change in transmission can be directly related to a shift in wavelength and consequently to the local field at the probe tip.

Scanning a probe in physical contact with a sample can lead to modifications of its surface, which can severely affect the properties of photonic crystal ( $\mathrm{PhC}$ ) microcavities (MCs). Such modifications can be minimized by applying the tapping mode operation. This, however, is more complicated, because it implies a strong nonlinear periodic variation of the interaction strength of the probe with the evanescent field. The resulting pattern as obtained from a slow photodiode is dependent on the tapping amplitude.

In this paper we will explain the method, show some results for a Fabry-Perot-like cavity resonator in a photonic crystal slab, discuss the interpretation of the results, and show a tapping mode result obtained using the T-SNOM method.

\section{Design and T-SNOM setup}

The experiments reported in this paper were performed on a PhC MC design in SOI $(220 \mathrm{~nm}$ device layer thickness on $1 \mu \mathrm{m}$ buried oxide) in a triangular lattice of holes with a radius of $270 \mathrm{~nm}$ and a periodicity of $440 \mathrm{~nm}$. For practical purposes we designed a relatively large Fabry-Perot-like cavity $(\sim 2 \mu \mathrm{m})$, see Fig. 1a. This high-finesse cavity has a $Q$ of about 650, see Fig. 1b. This $Q$ value was sufficient for our purpose, showing already a strong interaction of the probe with the resonator, although much higher $Q$ 's can be attained if needed, by optimizing the cavity design [5]. For feeding the PhC cavity we exploited W1 waveguides (i.e. one row of holes left out). The connecting photonic wires (rib-waveguides) had a width of $600 \mathrm{~nm}$, which ensures single TE-mode operation for wavelengths around $1550 \mathrm{~nm}$. The structure as shown in the SEM photo in Fig. 1a, was fabricated (at IMEC, Belgium) using a process [6], involving deep UV lithography $(\lambda=248 \mathrm{~nm})$ and reactive ion etching. A resonance wavelength of $1539.25 \mathrm{~nm}$ was measured, see Fig. 1b.

This work was supported by NanoNed, a national nanotechnology program coordinated by the Dutch ministry of Economic Affairs, and was also supported by the European Network of Excellence ePIXnet. 


\section{IWF7.pdf}

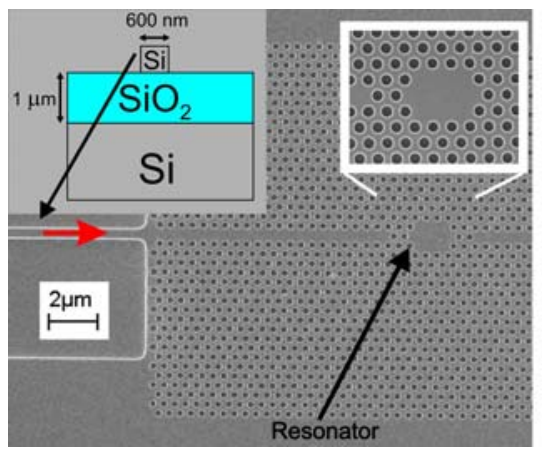

(a)

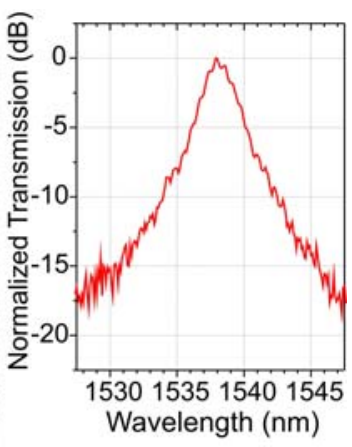

(b)

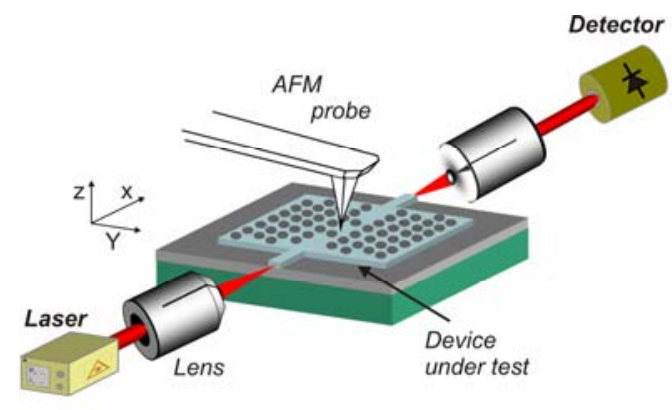

(c)

Fig. 1. (a) SEM image of the structure, a Fabry-Perot-type cavity formed between extra holes in a W1 waveguide in a photonic crystal slab. (b) Transmission spectrum of the undisturbed device around a resonance wavelength $(Q \cong 650)$. (c) T-SNOM set-up. Light from a tuneable laser is coupled into the device under test using a microscope objective. The transmitted light is collected on a photodetector, and the transmission is recorded for each position of the scanning AFM tip.

The measurement setup is shown in Fig. 1c. The light transmission through the device under test is recorded for each position of the AFM-tip, which is raster-scanned over the surface. The strongest interaction can be expected at those positions where the optical field has the largest intensity, which allows for mapping out the optical field distribution.

The AFM probe can be scanned across the device either in contact mode or in tapping mode, as mentioned in the section before. The highest sensitivity and resolution can be expected in contact mode, however damage to both the tip and the sample is evident after multiple scans. In tapping mode (driving the cantilever at an oscillation frequency of $63 \mathrm{kHz}$ ), damage is much less likely to occur, but resolution and sensitivity may be less because of the reduced interaction with the evanescent field of the resonance.

\section{Contact mode T-SNOM measurements}

In Fig. 3a we present the geometry data obtained from the AFM. The optical transmission that is recorded for each tip-position is shown in Fig. 3b. By combining both images and enhancing the display of the hole positions, we derive the "optical field distribution" within the resonator, see Fig.3c.

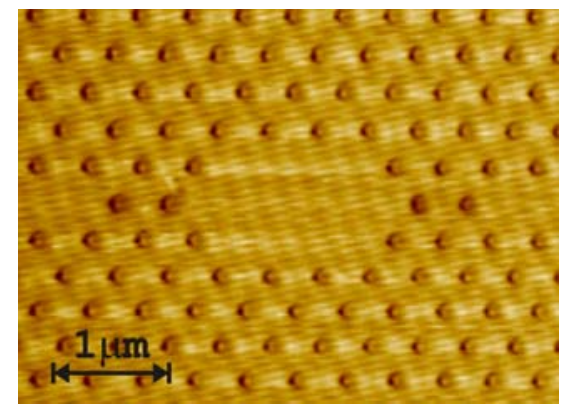

(a)

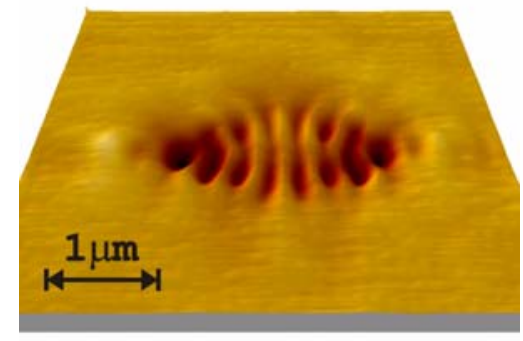

(b)

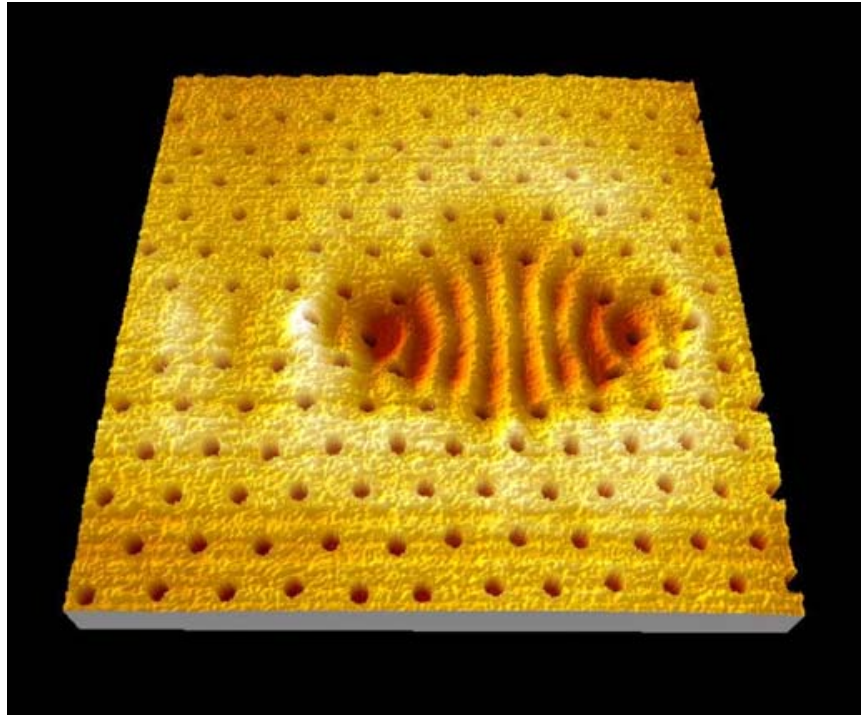

(c)

Fig. 3: Image formed by scanning an AFM tip over the top surface of the resonator structure. (a) Height data (conventional AFM image). (b). Optical transmission versus AFM tip position (T-SNOM image). (c) Superposition of images (a) and (b), showing the location of interaction maxima relative to the structural device features (the holes are enhanced for clarity). 


\section{IWF7.pdf}

For this experiment we exploited a $\mathrm{Si}_{3} \mathrm{~N}_{4}$ tip with an initial minimum radius of $10 \mathrm{~nm}$ in contact mode. As can be seen in Fig. 3c, the T-SNOM method is capable of mapping out submicrometer patterns. From the optical data, we estimate a resolution for the method smaller than $100 \mathrm{~nm}$.

\section{Tapping mode T-SNOM measurements}

The tapping-mode experiments were performed using a Si probe with an initial radius of $7.5 \mathrm{~nm}$. The results for a tapping amplitude of $53 \mathrm{~nm}$ is shown in Fig. 4a. The hole pattern has been overlaid to show where the highest interaction is found.

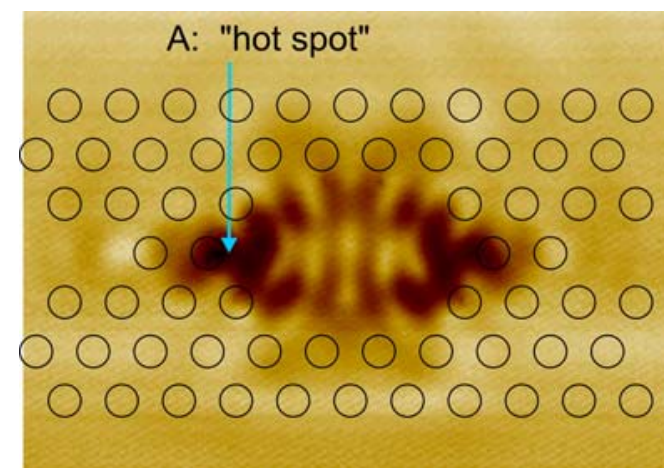

(a)

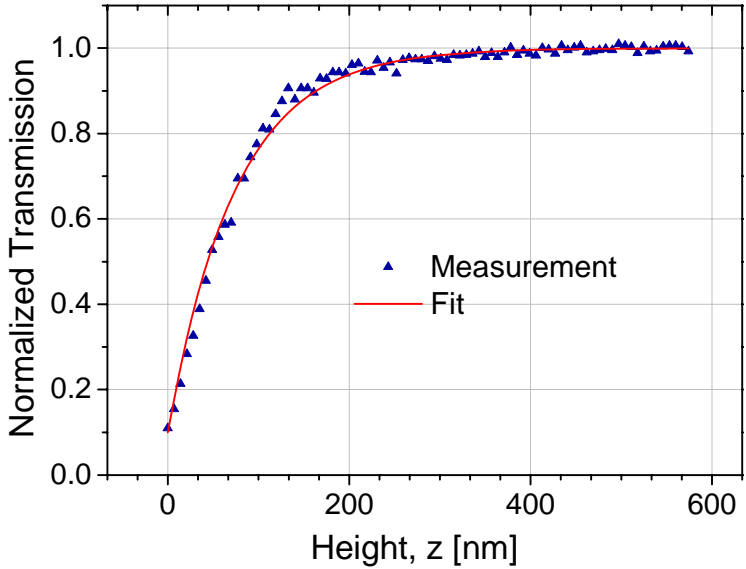

(b)

Fig. 4. (a) Tapping T-SNOM images with the mask pattern overlaid to indicate the position of the holes. The tapping amplitude was $53 \mathrm{~nm}$ (b) At the position denoted by "A" in the left figure, we have measured the tip-height dependent transmission $T(z)$, an exponential curve, $T(z)=1-0.9 * \exp (-(z / 74.5))$, has been fitted to the data points.

The strongest interaction is found at the hot spot indicated by the letter A. The cavity can be well manipulated at this position to achieve tuning of the resonance wavelength or quality factor. The transmission versus tip height at spot A can be well approximated by an exponential curve as can be expected from the exponential decaying evanescent field (Fig. 4b). If combined with a simple model for the tip dynamics, this curve can be used to predict the mechanooptical interaction as a function of the tapping amplitude as measured on a slow detector (maximum response frequency $<<63 \mathrm{kHz}$ ).

The curve in Fig. $4 \mathrm{~b}$ shows that displacements in the order of $100 \mathrm{~nm}$ have a strong optical effect, which demonstrates the possibility of developing nanomechano-optically actuated tunable filters or switches. Due to the small required displacement of small a mass, such devices can be expected to operate at much higher speed and lower (stationary) driving power than conventional mechano-optical or thermo-optical ones.

\section{Conclusions}

A recently developed optical characterization technique, T-SNOM, that is especially suited for optical microresonators has been demonstrated by applying it to a photonic crystal microcavity. The results show the method to be very appropriate for determining the field distribution of a microcavity at resonance. Although contact mode experiments provide the highest accuracy, they may damage both the AFM-tip and the device. The tapping mode has been demonstrated to be a viable alternative.

\section{References}

[1] I. Märki, M. Salt, and H. P. Herzig, "Tuning the resonance of a photonic crystal microcavity with an AFM probe”, Opt. Express 14, 2969$2978(2006)$

[2] W. C. L. Hopman, A. J. F. Hollink, R. M. De Ridder, K. O. Van Der Werf, V. Subramaniam, and W. Bogaerts, "Nano-mechanical tuning and imaging of a photonic crystal micro-cavity resonance", Opt. Express 14, 8745-8752 (2006).

[3] J. T. Robinson, S. F. Preble, and M. Lipson, "Imaging highly confined modes in sub-micron scale silicon waveguides using transmissionbased near-field scanning optical microscopy", Opt. Express 14, 10588-10595 (2006).

[4] W. C. L. Hopman, K. O. van der Werf, A. J. F. Hollink, W. Bogaerts, V. Subramaniam, and R. M. de Ridder, "Experimental verification of a simple transmission model for predicting the interaction of an AFM-probe with a photonic crystal micro-cavity in tapping mode operation", Submitted for publication, (2007).

[5] Y. Akahane, T. Asano, B. S. Song, and S. Noda, "High-Q photonic nanocavity in a two-dimensional photonic crystal", Nature 425, 944-947 (2003).

[6] W. Bogaerts, R. Baets, P. Dumon, V. Wiaux, S. Beckx, D. Taillaert, B. Luyssaert, J. Van Campenhout, P. Bienstman, and D. Van Thourhout, "Nanophotonic waveguides in silicon-on-insulator fabricated with CMOS technology", J. Lightwave Technol. 23, 401-412 ( 2005). 